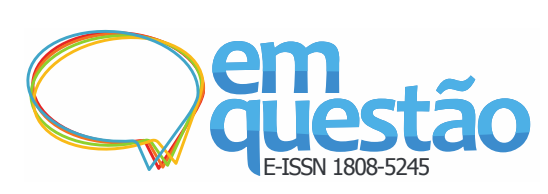

\title{
Contribuição da estatística multivariada para a avaliação dos programas de pós-graduação no Brasil: um estudo na área da Ciência da Informação
}

\author{
Renata Cristina Gutierres Castanha \\ Doutoranda; Universidade Estadual Paulista "Júlio de Mesquita Filho", Marília, SP, Brasil; \\ regutierres@gmail.com \\ Maria Cláudia Cabrini Grácio \\ Doutora; Universidade Estadual Paulista "Júlio de Mesquita Filho", Marília, SP, Brasil; \\ cabrini@marilia.unesp.br
}

\begin{abstract}
Resumo: Esta pesquisa tem como objetivo geral analisar a contribuição de técnicas estatísticas uni-multivariadas como um método de suporte ao entendimento da complexa metodologia de avaliação de programas de pósgraduação realizada pela Coordenação de Aperfeiçoamento de Pessoal de Nível Superior. De forma mais específica, esta pesquisa busca identificar os agrupamentos dos programas de pós-graduação em Ciência da Informação, gerados pela análise de cluster e os dados gerados pelos gráficos boxplot, com base no conjunto de indicadores presentes na Planilha Comparativa da Avaliação Trienal da área. Posteriormente, comparou-se os grupos de programas de pósgraduação, segundo a nota obtida pela avaliação com o resultado do agrupamento obtido pela análise de cluster, a fim de visualizar a participação e suficiência dos indicadores na atribuição dos conceitos dos programas de pós-graduação. Para os 14 programas de pós-graduação da área de Ciência da Informação, no triênio 2013, analisaram-se os seguintes indicadores: total de teses e dissertações defendidas, média de artigos publicados segundo a estratificação Qualis, média de trabalhos completos publicados em anais de eventos técnico-científicos, livros, capítulos de livro, texto integral, coletâneas, verbetes e outros e produção artística, ponderados por docente permanente. De acordo com os resultados obtidos, observou-se que, embora os agrupamentos formados apresentem conceitos próximos, apenas os indicadores presentes na Planilha não são suficientes para discerni-los, sendo necessária a inclusão de indicadores qualitativos, uma vez que os indicadores de produção não são suficientes para distinguir os programas de acordo com seus conceitos.
\end{abstract}

Palavras-chave: Avaliação da pós-graduação. Análise de cluster. Indicadores bibliométricos. Pós-graduação em Ciência da Informação. 


\section{Introdução}

O sistema de avaliação dos programas de pós-graduação brasileiro vem sendo continuamente aperfeiçoado e constitui instrumento de orientação tanto para a comunidade científica como para os próprios programas de pós-graduação, os quais procuram um padrão de excelência acadêmica nacional.

Os resultados da avaliação realizada pela Coordenação de Aperfeiçoamento de Pessoal de Nível Superior (CAPES) contribuem para o estabelecimento de políticas científicas relativas à pós-graduação stricto sensu, bem como para a aplicação das ações de fomento no país. Em decorrência da relevância desse sistema de avaliação, destaca-se a importância da sua análise e discussão e do desenvolvimento das metodologias que contribuam para seu refinamento (COORDENAÇÃO..., 2014).

Para avaliar o desempenho dos programas de pós-graduação brasileiros, a CAPES adota cinco quesitos, com pesos diferentes na composição da nota final: proposta do programa (1); corpo docente (2); corpo discente, teses e dissertações (3); produção intelectual (4); e inserção social (5). Cada quesito apresenta de três a cinco itens de avaliação, com pesos diferentes dentro do quesito. Em todos os quesitos, a soma dos pesos dos itens de avaliação é igual a 100. Com base nos cinco quesitos de avaliação apresentados, a CAPES atribui notas de 1 a 7 aos programas de pós-graduação brasileiros.

Os cursos com notas 1 e 2 são descredenciados pela CAPES; a nota 3 é atribuída para programas com o padrão mínimo de qualidade; a nota 4, para aqueles com bom desempenho; e a nota 5, para cursos com alto nível de desempenho. Notas 6 e 7 são atribuídas aos programas que apresentem desempenho equivalente ao dos centros internacionais de excelência, que tenham um nível de desempenho altamente diferenciado e de liderança nacional em relação aos demais programas da área (COORDENAÇÃO..., 2014).

Os quesitos com maior peso na composição final da nota de um programa de pós-graduação são os quesitos "Corpo discente, teses e dissertações" e "Produção intelectual": juntos compõem $70 \%$ do peso na nota final. O quesito 3, 
"Corpo discente, teses e dissertações", é composto por quatro itens de avaliação, sendo os dois primeiros itens com maior peso, no total de 30 cada item, a saber: quantidade de teses e dissertações defendidas no período de avaliação e qualidade das teses e dissertações e da produção de discentes autores da pós-graduação e da graduação na produção científica do programa, aferida por publicações e outros indicadores pertinentes à área.

Os dois itens seguintes têm o segundo maior peso (igual a 20, cada item) nesse quesito, a saber: tempo médio de titulação de mestres e doutores, especialmente de bolsistas; e quantidade de teses e dissertações defendidas no período de avaliação, em relação ao corpo docente permanente e à dimensão do corpo discente.

O quesito 4 - "Produção intelectual" - é composto por quatro itens de avaliação. O item com maior peso (igual a 65) nesse quesito é constituído pela média ponderada das produções qualificadas em periódicos, livros e capítulos dos docentes permanentes, bem como em trabalhos completos em anais (COORDENAÇÃO..., 2014).

Nesse sentido, é importante destacar a contribuição dos estudos bibliométricos como metodologia objetiva para a análise do desempenho da pós graduação brasileira, ao se trabalhar com indicadores relativizados às particularidades, especificidades e tendências das diferentes áreas do conhecimento científico.

Para que tais indicadores possam ser devidamente interpretados e contextualizados, são necessários conhecimentos oriundos de estudos históricos e epistemológicos, entre outros, os quais permitem conhecer as tradições, os paradigmas, assim como os documentos e formas de expressão e influências mútuas em relação aos diferentes campos e domínios científicos, permitindo uma perspectiva mais profunda e coerente quando se busca entender os documentos, a organização, os sistemas, a informação e o conhecimento (CASTANHA, 2014; HJØRLAND, 2002).

Entre os procedimentos bibliométricos, destacam-se as análises multivariadas para os estudos destinados ao aperfeiçoamento da metodologia de avaliação dos programas de pós-graduação, uma vez que essas análises 
consideram simultaneamente e de forma articulada os indicadores quantitativos armazenados e utilizados pela CAPES em seu sistema de avaliação.

Em especial, destaca-se a análise de cluster, método utilizado nesta pesquisa para a análise do desempenho dos programas de pós-graduação da área de Ciência da Informação, ao possibilitar a taxonomia dos programas de pósgraduação, ao agrupá-los de forma que aqueles pertencentes a um mesmo agrupamento sejam semelhantes entre si em relação aos indicadores quantitativos utilizados pela CAPES, e os programas de pós-graduação em agrupamentos distintos sejam heterogêneos em relação aos mesmos indicadores.

A análise de cluster é destinada ao estudo das relações de interdependência entre um conjunto de variáveis interdependentes, permitindo agrupar elementos segundo suas similaridades e proximidades em relação a esse conjunto de variáveis (HAIR et al., 2009).

Desse modo, os elementos em cada cluster tendem a ser semelhantes entre si e dissemelhantes dos outros elementos presentes em outros clusters. Nesse sentido, considera-se que a aplicação desse método ao conjunto de indicadores dos programas de pós-graduação possibilita a visualização do agrupamento daqueles, em função das semelhanças identificadas entre eles, pela análise simultânea e multivariada dos indicadores de avaliação que são utilizados pela CAPES.

Isso posto, esta pesquisa tem como objetivo geral analisar a contribuição da análise de cluster como método que auxilie no desenvolvimento e aperfeiçoamento da complexa metodologia de avaliação de programas de pósgraduação realizada pela CAPES, a partir dos indicadores relativos à área de Ciência da Informação referentes ao triênio 2013, o qual compreende o período de 2010 a 2012.

De forma mais específica, busca identificar e evidenciar os agrupamentos dos programas de pós-graduação em Ciência da Informação, gerados pela análise de cluster, com base no conjunto de indicadores presentes na Planilha Comparativa da Avaliação Trienal da área (COORDENAÇÃO..., 2013b). Ainda, pretende comparar os grupos de programas de pós-graduação segundo o conceito atribuído pela avaliação da CAPES, com o resultado do agrupamento obtido por 
meio da análise de cluster, a fim de visualizar a participação e suficiência dos indicadores na atribuição dos conceitos final dos programas de pós-graduação.

No que se refere aos cursos stricto sensu na área de Ciência da Informação, os mais antigos tiveram início na década de 1970 e durante quase 40 anos os programas vêm passando por um processo constante e regular de desenvolvimento e amadurecimento. A área de Ciência da Informação integra a grande área da CAPES de Ciências Sociais Aplicadas I, a qual é formada por três campos de conhecimento, Comunicação, Ciência da Informação e Museologia, e reúne, ao todo, 89 programas de pós-graduação (COORDENAÇÃO..., 2013a).

\section{Procedimentos metodológicos}

Inicialmente, foram identificados apenas os programas de pós-graduação caracterizados como Ciência da Informação e afins, da modalidade Mestrado e Doutorado Acadêmicos, utilizando-se como fonte de dados a Planilha Comparativa da Avaliação Trienal da área de Ciências Sociais Aplicadas I (COORDENAÇÃO..., 2013b), referente à avaliação do último triênio (20102012), realizada em 2013. Optou-se pela escolha apenas de programas de modalidade Mestrado e Doutorado Acadêmicos, uma vez que a ponderação de seus indicadores difere dos demais programas de Mestrado Profissional.

$\mathrm{Na}$ planilha em questão, consta um total de 89 programas de pósgraduação na grande área de Ciências Sociais Aplicadas I, dos quais foram utilizados para a análise 14, sendo os programas de pós-graduação em Ciência da Informação das universidades a seguir: Universidade Estadual de Londrina (UEL), Universidade Federal da Bahia (UFBA), Universidade Federal Fluminense (UFF), Universidade Federal da Paraíba (UFPB), Universidade Federal de Pernambuco (UFPE), Universidade Federal de Santa Catarina (UFSC), Universidade de Brasília (UNB), Instituto Brasileiro de Informação em Ciência e Tecnologia (IBICT - UFRJ), Universidade de São Paulo (USP), Universidade Federal de Minas Gerais (UFMG) e Universidade Estadual Paulista (UNESP/Marília), além dos programas da Universidade de São Paulo (USP Museologia), Universidade Federal do Estado do Rio de Janeiro (UNIRIO - 
Museologia e Patrimônio) e Universidade Federal do Rio Grande do Sul (UFRGS - Comunicação e Informação).

A partir do conjunto de indicadores da Planilha Comparativa da Avaliação Trienal da área (COORDENAÇÃO..., 2013b), foram extraídos os indicadores quantitativos de cada programa, a saber: total de teses e dissertações defendidas, média de artigos por docente permanente, segundo a estratificação Qualis, e média das demais produções bibliográficas.

Os indicadores de produção científica foram ponderados seguindo a pontuação por estrato Qualis, relativizados em função do total de docentes permanentes de cada programa e divididos por três, o que corresponde ao triênio em que foram analisados, a fim de se eliminar vieses em função do tamanho dos programas de pós-graduação em Ciência da Informação.

Posteriormente, calcularam-se os índices qualitativo e qualiquantitativo, característicos da área Ciências Sociais Aplicadas I. O índice qualiquantitativo do programa no triênio é obtido pela divisão da média de produção anual por docente pela mediana (ponto médio entre o maior valor e o menor valor das produções) dessa produção, e o índice qualitativo é a média ponderada obtida pelo total de pontos obtidos pelo Programa dividido pelo número de itens publicados.

Utilizou-se o software SPSS para a construção dos gráficos boxplot e para a análise de clusters, esta desenvolvida utilizando o método Ward, medida de distância euclidiana sem variáveis padronizadas e como variáveis os indicadores citados anteriormente. O resultado dessa análise foi apresentado em forma de dendrograma.

\section{Análise dos resultados}

A Figura 1 apresenta o dendrograma que expressa os agrupamentos dos programas de pós-graduação em Ciência da Informação do Brasil em função das suas similaridades relativas aos indicadores presentes na Planilha Comparativa da Avaliação Trienal da área (COORDENAÇÃO..., 2013b), referente ao triênio 2010-2012, em que se observa a constituição de quatro agrupamentos distintos denominados G1, G2, G3 e G4. 
O primeiro grupo (G1) é constituído por dois programas de pós-graduação com conceito 3. O segundo grupo (G2) é composto por sete programas de pósgraduação, sendo seis com conceito 4 e um com conceito 5. O terceiro grupo (G3) é composto por dois programas com conceitos 5 e 6, e o quarto grupo (G4), com três programas, apresenta conceitos 4,5 e 6 .

Nota-se que, com exceção do G1, os grupos restantes apresentam características heterogêneas, ou seja, os programas não se agruparam seguindo o padrão de apenas um único conceito, e, sim, de conceitos variados, todavia próximos: G1 - dois programas com conceito 3; G2 - programas com conceitos 4 e 5; G3 - programas com conceitos 5 e 6; G4 - programas com conceitos 4, 5 e 6 . Destaca-se também que dentro da área da Ciência da Informação, nenhum programa possui conceito 7 .

Figura 1 - Agrupamentos dos cursos de pós-graduação (G1, G2, G3 e G4) em Ciência da Informação, em função dos indicadores analisados: total de teses e dissertações defendidas, média de artigos por docente permanente segundo a estratificação Qualis, e média das demais produções bibliográficas.

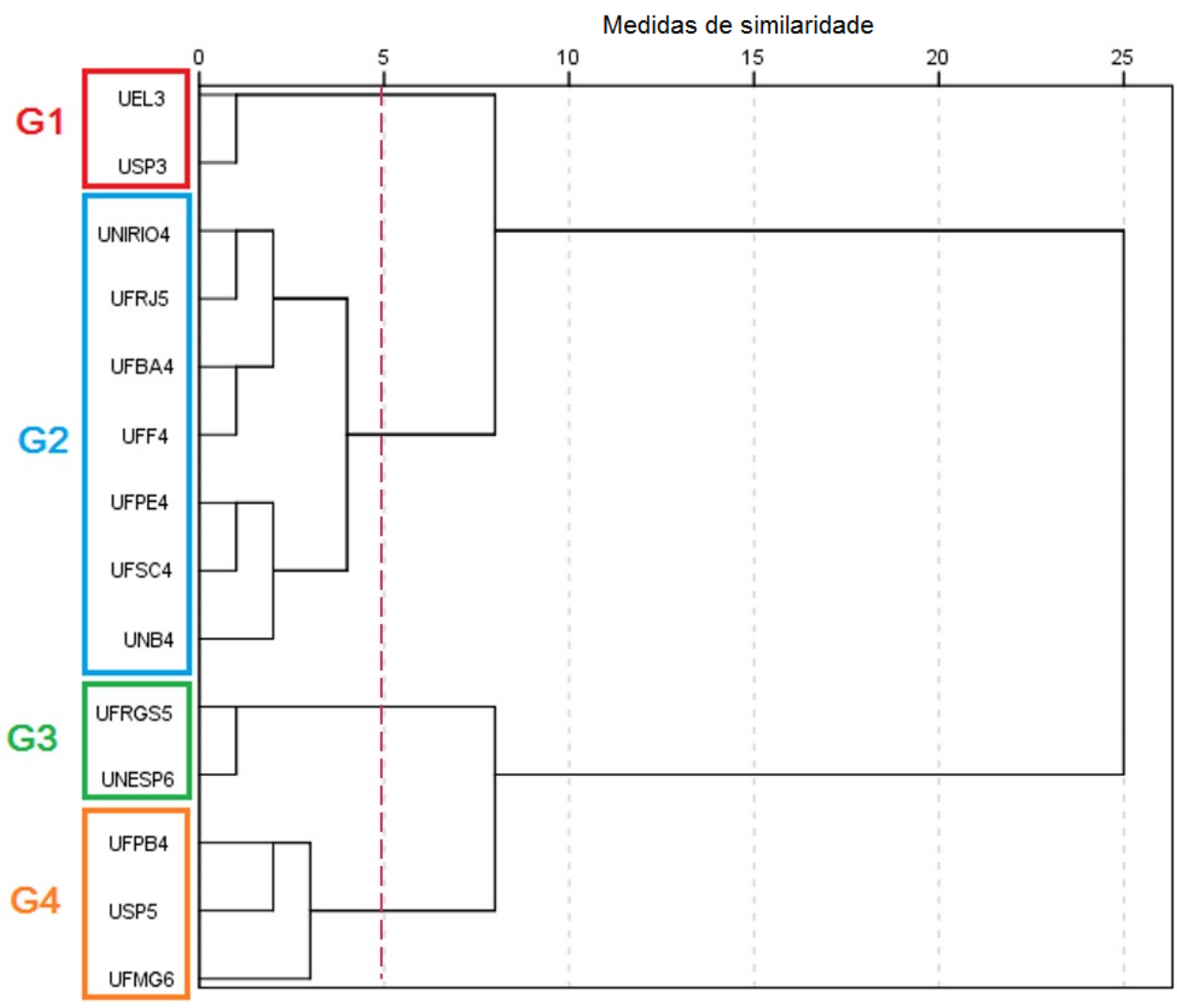

Fonte: Elaborado pelas autoras. 
Em relação ao G2, dentre os sete programas agrupados, a UFRJ é a única a apresentar conceito 5. Tem-se por hipótese que esse comportamento pode ser decorrente do baixo total de teses defendidas no período e do fato de os programas com conceito 4 não terem teses defendidas, e das produções bibliográficas apresentarem valores menores que os demais programas com conceito 5, em geral.

Em relação ao G3, os programas agrupados foram UFRGS com conceito 5 e UNESP/Marília com conceito 6. A razão de esses programas estarem tão próximos se deve, provavelmente, às similaridades de publicações dos mesmos, uma vez que ambos apresentam alta produtividade em relação ao total de dissertações e teses, assim como a algumas tipologias da produção bibliográfica, o que parece ser característico da área.

O G4 apresenta comportamento similar ao G3, uma vez que os programas agrupados, UFPB, USP e UFMG, apesar de apresentarem conceitos distintos, 4, 5 e 6 respectivamente, apresentam comportamentos próximos em relação às produções bibliográficas e também em relação às dissertações e teses.

Para uma visualização mais completa do comportamento dos programas de pós-graduação em Ciência da Informação em relação aos indicadores analisados, construíram-se gráficos boxplot com os indicadores oriundos da Planilha Comparativa da Avaliação Trienal (COORDENAÇÃO..., 2013b), por estrato de conceito atribuído pela CAPES no triênio. A Figura 2 apresenta o gráfico boxplot referente ao total de teses e dissertações defendidas no período em relação ao conceito dos programas.

Observa-se uma tendência ascendente entre o total de teses defendidas e o conceito dos programas, ou seja, partindo do conceito 3 para o 6 , quanto menor o conceito do programa, menos defesas este apresenta ao longo do período analisado em relação aos programas com maiores conceitos, comportamento que já é esperado.

Em relação às dissertações, os programas com conceitos entre 4 e 6 apresentam comportamento semelhante, embora quantitativamente diferentes. Programas com conceito 3 não apresentaram teses e dissertações defendidas no período, e programas com conceito 4 apresentaram apenas dissertações, uma vez 
que esse conceito é atribuído a programas de pós-graduação que estão implementando o doutorado.

No estrato do conceito 4, destaca-se como outlier superior o programa da UNB, que, apesar de apresentar conceito 4, apresenta um total de teses defendida no período muito maior do que todos os outros programas com mesmo conceito.

Ainda, a mediana relativa às dissertações apresenta uma assimetria com deslocamento em direção ao primeiro quartil, o que significa que há um número considerável de programas de pós-graduação apresentando um total de defesas no período inferior ao restante dos programas com mesmo conceito, e, ainda, observa-se muita variação em relação ao total de publicação entre eles.

Figura 2 - Gráfico boxplot referente ao total de teses e dissertações defendidas no período, relativizadas em função do total de docentes permanentes e do triênio, em relação ao conceito dos programas de pós-graduação em Ciência da Informação.

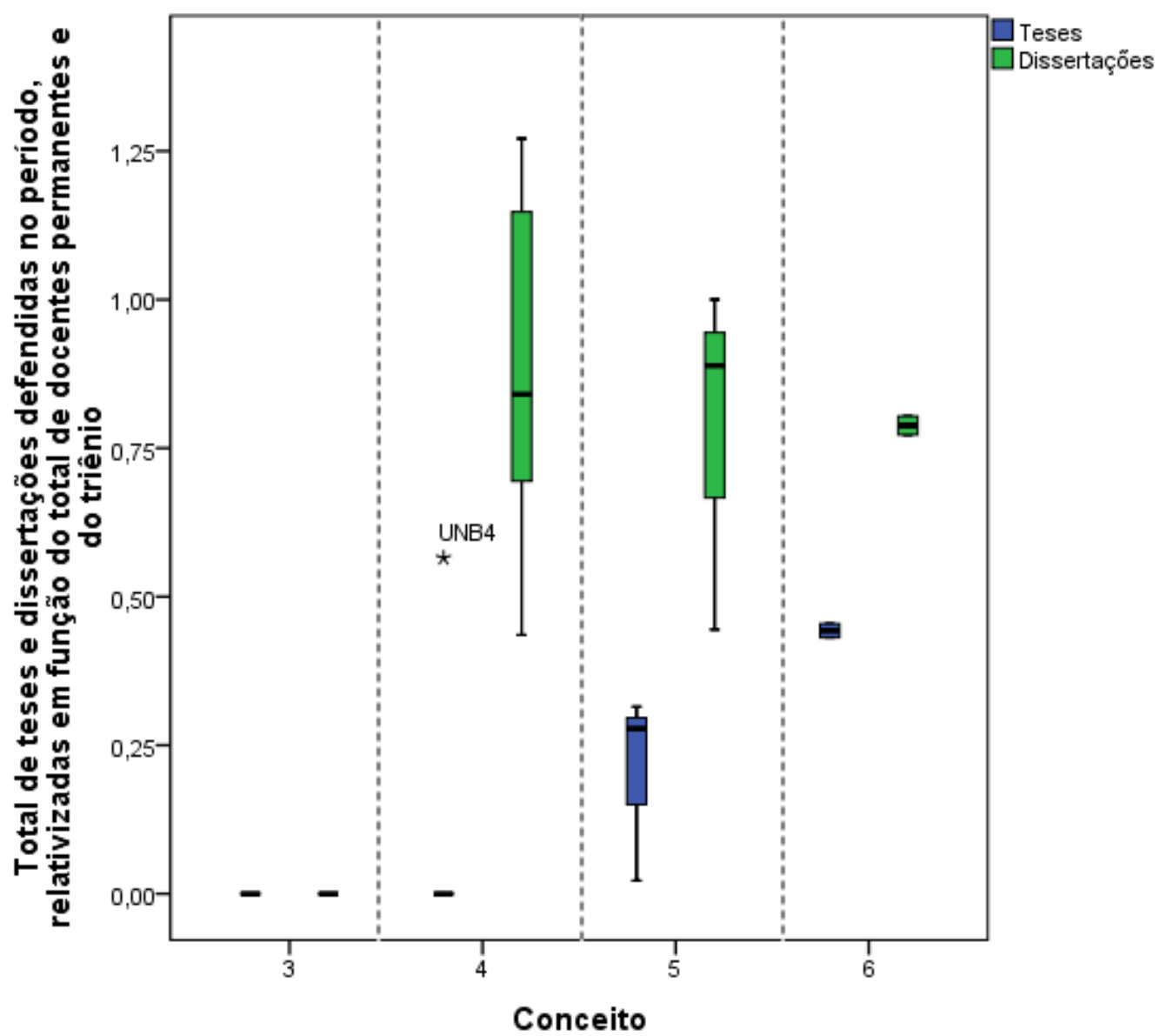

Fonte: Elaborado pelas autoras.

Já no estrato com conceito 5, a mediana relativa às dissertações apresenta uma assimetria com deslocamento em direção ao terceiro quartil, o que significa 
que há um número considerável de programas de pós-graduação apresentando um total de defesas no período superior ao restante dos programas com mesmo conceito, e, ainda, muita variação em relação ao total de publicação entre eles.

A Figura 3 apresenta o gráfico boxplot referente ao total de artigos publicados em periódicos, por docente, no período em relação ao conceito dos programas.

Figura 3 - Gráfico boxplot referente ao total de artigos publicados em periódicos Qualis A1, A2, B1, B2, B3, B4 e B5, relativizados em função do total de docentes permanentes e do triênio em relação ao conceito dos programas de pós-graduação em Ciência da Informação.

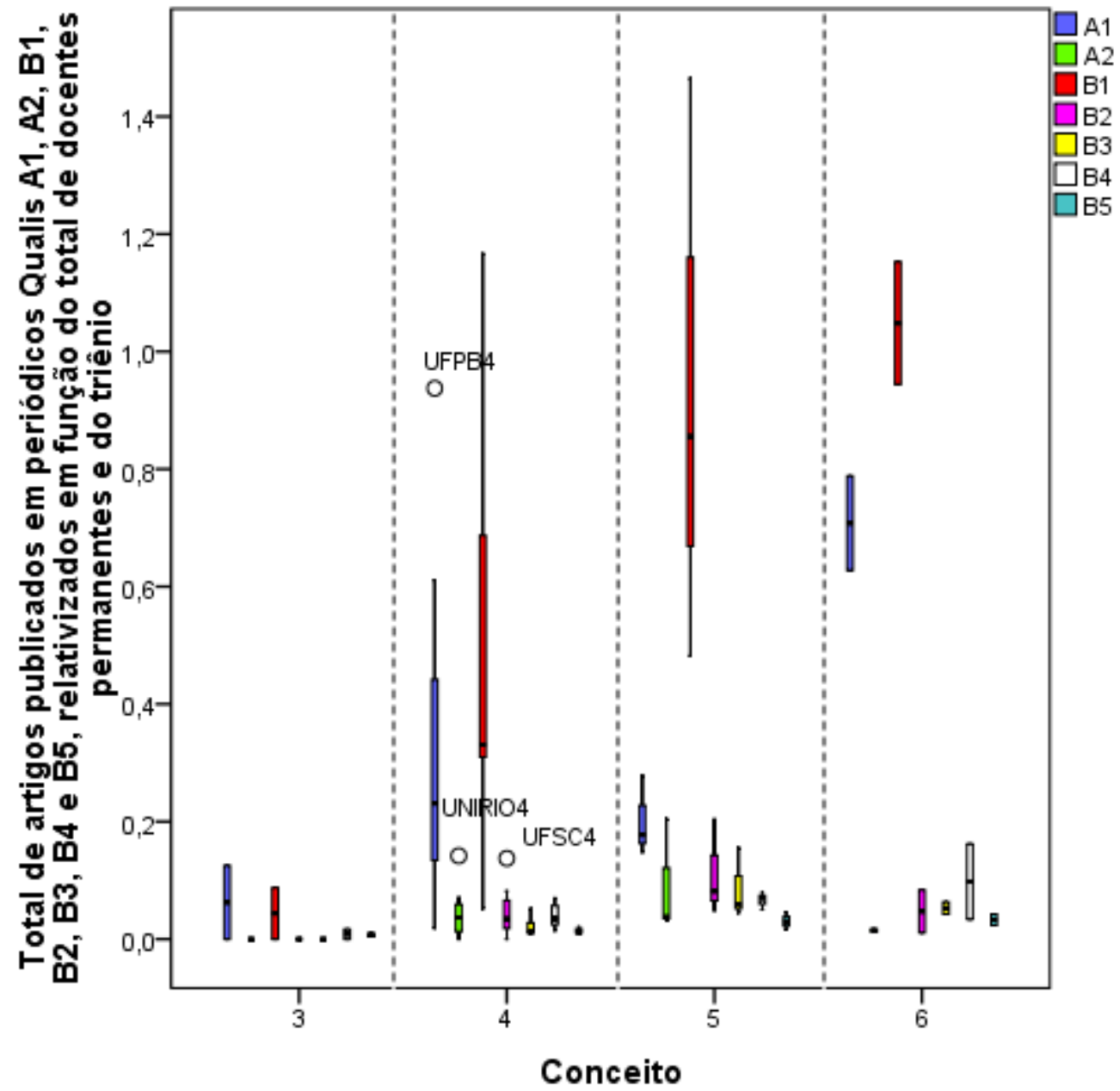

Fonte: Elaborado pelas autoras.

Em geral, os programas apresentaram um total de publicações por docente superior nos periódicos Qualis B1. Dessa maneira, é possível observar uma tendência ascendente entre o total de artigos publicados em periódicos Qualis B1, por docente, e o conceito dos programas, ou seja, partindo do conceito 3 para o 6 , 
quanto menor o conceito do programa, menos artigos publicados este apresenta, ao longo do período analisado, em relação aos programas com maiores conceitos.

Em relação ao conceito 4, este apresenta um número considerável de publicações em periódicos Qualis A1 e B1, com destaque para os outliers superiores da UFPB. Em relação ao Qualis A2 e B3, destaca-se a UNIRIO como outlier superior.

Ainda em relação ao conceito 4 , a mediana relativa às publicações A1 apresenta uma assimetria com deslocamento em direção ao primeiro quartil, o que significa que há um número considerável de programas de pós-graduação apresentando um total de publicações em periódicos Qualis A1 inferior ao restante dos programas com mesmo conceito, no período analisado, ao passo que a mediana relativa às publicações B1 apresenta uma assimetria com deslocamento em direção ao terceiro quartil, o que significa que há um número considerável de programas de pós-graduação apresentando um total de publicações em periódicos Qualis B1 superior ao restante dos programas com mesmo conceito.

Em relação aos conceitos 5 e 6 , parece haver um comportamento semelhante de publicação em relação à publicação em periódicos Qualis B1, embora os programas com conceito 5 sejam quantitativamente inferiores em relação às publicações em periódicos Qualis A1. Esse parece ser um comportamento característico da área, uma vez que esta possui um maior número de periódicos indexados Qualis B1 do que o restante.

A Figura 4 apresenta o gráfico boxplot referente ao total de trabalhos completos publicados em anais de eventos técnico-científicos, capítulos de livro e texto integral, coletâneas, verbetes e outros e produção artística, por docente, no período, em relação ao conceito dos programas.

Observa-se uma tendência ascendente entre o total de publicações em trabalhos completos em anais de eventos técnico-científicos e capítulos de livro e o conceito do programa, ou seja, partindo do conceito 3 para o 6, quanto menor o conceito do programa, menos defesas este apresenta ao longo do período analisado, em relação aos programas com maiores conceitos.

Todos os programas analisados apresentam comportamento semelhante, embora quantitativamente diferentes, o que parece ser característico da área, uma 
vez que esta apresenta eventos, cujos registros bibliográficos dos trabalhos apresentados são disseminados em livros em lugar de atas, proceedings ou anais de eventos.

Figura 4 - Gráfico boxplot referente ao total de trabalhos completos publicados em anais de eventos técnico-científicos, capítulos de livro e texto integral, coletâneas, verbetes e outros e produção artística, relativizados em função do total de docentes permanentes e do triênio em relação ao conceito dos programas de pós-graduação em Ciência da Informação.

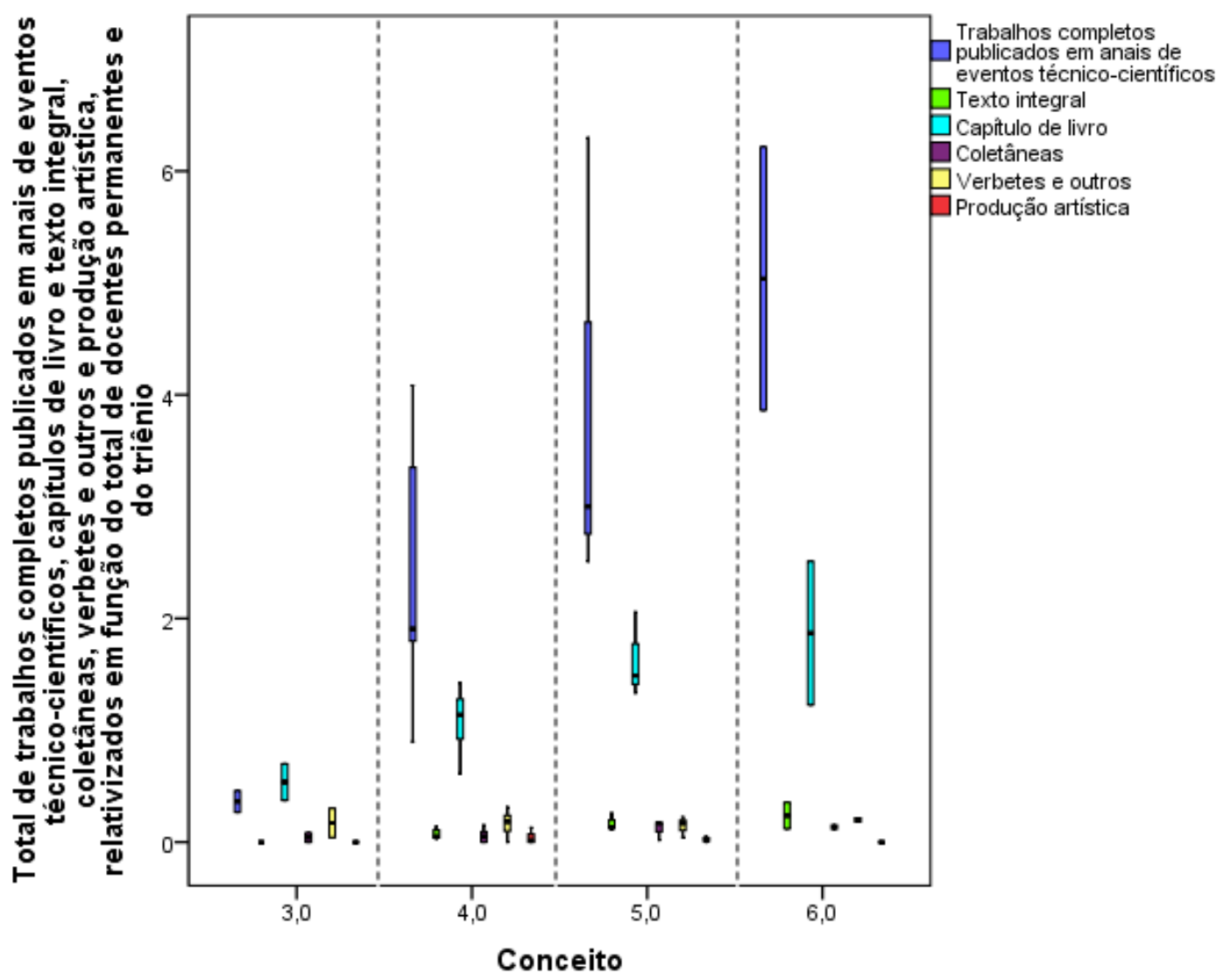

Fonte: Elaborado pelas autoras.

Especificamente em relação ao conceito 4, a mediana relativa ao total de publicações em trabalhos completos publicados em anais de eventos técnicocientíficos apresenta uma assimetria com deslocamento em direção ao primeiro quartil, o que significa que há um número considerável de programas de pósgraduação apresentando quantidade de publicações de trabalhos completos em anais de eventos técnico-científicos inferior ao restante dos programas com mesmo conceito, no período analisado, ao passo que a mediana relativa às publicações em capítulos de livro apresenta uma assimetria com deslocamento em 
direção ao terceiro quartil, o que significa que há um número considerável de programas de pós-graduação apresentando um total de publicações em capítulos de livro superior ao restante dos programas com mesmo conceito.

Em relação ao conceito 5, a mediana relativa ao total de publicações em trabalhos completos em anais de eventos técnico-científicos apresenta uma assimetria com deslocamento em direção ao primeiro quartil, o que significa que há um número considerável de programas de pós-graduação apresentando um total de publicações de trabalhos completos publicados em anais de eventos técnico-científicos inferior ao restante dos programas com mesmo conceito, no período analisado, congruente com a mediana relativa às publicações em capítulos de livro, a qual apresenta uma assimetria com deslocamento em direção ao primeiro quartil, o que significa que há um número considerável de programas de pós-graduação apresentando um total de publicações em capítulos de livro inferior ao restante dos programas com mesmo conceito.

\section{Considerações finais}

De acordo com todos os indicadores analisados, observou-se a formação de um grupo homogêneo e dois grupos heterogêneos no dendrograma. G2 mescla os conceitos 4 e 5, G3 mescla os conceitos 5 e 6 e G4 mescla os conceitos 4, 5 e 6 .

Esse comportamento sugere que, embora já propiciem uma significativa similaridade e proximidade com os conceitos atribuídos pela CAPES, os indicadores quantitativos presentes na Planilha Comparativa de Avaliação não foram suficientes para agrupar os programas de forma idêntica à presente na avaliação da CAPES, necessitando assim também dos indicadores qualitativos, discriminados no quesito Proposta do Programa, em que se evidencia trajetória, metas, colaborações científicas, nucleação, solidariedade, internalização do conhecimento, vocação, entre outras, a fim de se obter um agrupamento exato dos programas. Desse modo, para uma avaliação mais refinada, considera-se necessária a inclusão de outros indicadores, que não estão presentes na Planilha Comparativa da Avaliação Trienal da área de Ciências Sociais Aplicadas I (COORDENAÇÃO..., 2013b) aqui analisada. 
Destaca-se a importância dos gráficos tipo boxplot para a análise do desempenho dos programas agrupados por conceito, uma vez que é possível observar a tendência geral de cada conceito quanto a cada indicador individualmente, em relação ao comportamento geral dos demais conceitos. Ainda, possibilita a visualização da presença de programas outliers (com desempenho discrepante em relação ao grupo a que pertence) e o desempenho destes em relação ao dos demais programas com o mesmo conceito, assim como em relação ao desempenho dos outros conceitos.

Observou-se, por meio da análise dos gráficos boxplot, que a Ciência da Informação apresenta uma tendência ascendente entre os seguintes indicadores: total de teses defendidas e o conceito dos programas, total de publicações em periódicos Qualis B1 por docente e o conceito dos programas e total de publicações completas em anais de eventos técnico-científicos e capítulos de livro e o conceito do programa, o que parece ser uma característica de publicação científica da área, uma vez que esta possui muitos programas com doutorado, além de a maioria dos periódicos indexados estarem presentes no estrato Qualis B1 e muitos eventos com os registros bibliográficos dos trabalhos apresentados disseminados em capítulos de livros em lugar de anais de eventos.

Esses resultados indicam que a avaliação dos programas de pós-graduação pela CAPES depende também de indicadores qualitativos, presentes nas informações enviadas pelos próprios programas, sugerindo que o papel dos indicadores quantitativos considerados pela CAPES depende da área de conhecimento, uma vez que Castanha (2014) demonstrou que os programas de pós-graduação em Matemática foram eficientemente agrupados, segundo seus conceitos, por meio do mesmo método de análise multivariada.

\section{Financiamento}

Trabalho financiado pelo Conselho Nacional de Desenvolvimento Científico e Tecnológico (CNPq). 


\title{
Referências
}

CASTANHA, R. C. G. Indicadores de avaliação de programas de pósgraduação no Brasil: uma análise multivariada na área de Matemática. 2014. 126 f. Dissertação (Mestrado em Ciência da Informação) - Faculdade de Ciência da Informação e Ciências, Universidade Estadual Paulista, Marília.

COORDENAÇÃO DE APERFEIÇOAMENTO DE PESSOAL DE NÍVEL SUPERIOR. Documento de área e comissão. [Brasília]: CAPES, 2013a. Disponível em: <http://www.avaliacaotrienal2013.capes.gov.br/documento-dearea-e-comissao>. Acesso em: 1 dez. 2016.

COORDENAÇÃO DE APERFEIÇOAMENTO DE PESSOAL DE NÍVEL SUPERIOR. Planilhas de indicadores. [Planilhas comparativas de avaliação trienal]. [Brasília]: CAPES, 2013b. Disponível em:

$<$ http://www.avaliacaotrienal2013.capes.gov.br/resultados/planilhascomparativas > Acesso em: $1 \mathrm{dez} .2016$.

COORDENAÇÃO DE APERFEIÇOAMENTO DE PESSOAL DE NÍVEL SUPERIOR. Sobre a avaliação. [Brasília]: CAPES, 2014. Disponível em: <http://capes.gov.br/avaliacao/sobre-a-avaliacao>. Acesso em: 1 dez. 2016.

HAIR, J. F. et al. Multivariate data analysis. 7. ed. Upper Saddle River: Prentice Hall, 2009.

HJØRLAND, B. Domain analysis in information science: eleven approaches traditional as well as innovative. Journal of Documentation, London, v. 58, n. 4, p. 422- 462, 2002.

\section{The multivariate statistics contribution for graduate assessment in Brazil: a study in Information Science.}

\begin{abstract}
This research aims to analyze the contribution of multivariate statistics as a method to support the understanding of the complex methodology of graduate assessment conducted by Coordination for the Improvement of Higher Education Personnel. More specifically, this research seeks to identify the cluster of graduate programs in Information Science generated by cluster analysis and the data generated by boxplot graphics, based on the set of indicators content in the Comparative Spreadsheets of Triennial Assessment of the area, and then comparing groups of graduate programs, according to the concept obtained by the Coordination for the Improvement of Higher Education Personnel assessment, with the result of the cluster obtained by cluster analysis, in order to verify the participation and adequacy of indicators in the allocation of concepts of graduate
\end{abstract}


programs. For all 14 graduate programs of Information Science area, evaluated in the last triennial years (2010-2012), the following indicators were analyzed: total of theses and dissertations, total of papers published, according to Qualis stratification, total of papers published in conference proceedings, books and chapters, full text, collections, and other entries and artistic production published, weighted by faculty member. According to the results, it was observed that while the formed clusters present similar concepts, only the indicators present in the Comparative Spreadsheets of Triennial Assessment were not sufficient to discern them, the inclusion of quality indicators being necessary, since the production indicators are not sufficient to distinguish the programs according to their concepts.

Keywords: Graduate assessment. Cluster analysis. Bibliometric indicators. Information Science graduate program.

Recebido em: 19/09/2016

Aceito em: 21/10/2016 